\title{
Preoperative Serum Alkaline Phosphatase and Clinical Outcome of Off-Pump Coronary Artery Bypass Surgery
}

\author{
Tae Kyong Kim, MD; Deok Man Hong, MD, PhD; Yoon Hyeong Choi, MD; \\ Chang-Hoon Koo, MD; Youn Joung Cho, MD; Jun-Bean Park, MD, PhD; \\ Kyung Woo Park, MD, PhD; Hyo-Soo Kim, MD, PhD; Yunseok Jeon, MD, PhD
}

\begin{abstract}
Background: Serum alkaline phosphatase (ALP) is related to vascular calcification. In a recent study on percutaneous coronary intervention (PCI) with a drug-eluting stent, higher ALP was associated with poor clinical outcomes in terms of mortality, myocardial infarction, and stent thrombosis. The aim of this study was to evaluate the relationship between preoperative ALP and clinical outcome of off-pump coronary artery bypass surgery (OPCAB).
\end{abstract}

\begin{abstract}
Methods and Results: We retrospectively enrolled and reviewed a total of 1,335 patients who underwent OPCAB. Patients were divided into tertiles based on preoperative serum ALP $(<60,60-76$, and $>76 \mathrm{IU} / \mathrm{L})$. As preoperative ALP increased, the HR of mortality remained constant after adjusting for confounders. On Cox proportional hazards regression analysis, there was no association between ALP and all-cause mortality. The adjusted HR for all-cause mortality for the middle tertile was 0.882 ( $95 \% \mathrm{Cl}$ : $0.592-1.314$, $\mathrm{P}=0.537)$, and $0.915(95 \% \mathrm{Cl}: 0.605-1.383, \mathrm{P}=0.672)$ for the highest tertile. In addition, no associations between ALP and cardiovascular mortality, myocardial infarction, revascularization, or major adverse cardiac events were found.
\end{abstract}

Conclusions: Unlike after PCI, high ALP is not related to adverse clinical events, such as mortality, myocardial infarction, or revascularization after OPCAB. ALP might be considered when determining the optimal revascularization technique.

Key Words: Alkaline phosphatase; Clinical outcome; Off-pump coronary artery bypass surgery

$\mathbf{T}$ he enzyme alkaline phosphatase (ALP) plays a critical role in bone mineralization. ${ }^{1}$ It catalyzes the hydrolysis of inorganic pyrophosphate, thereby decreasing this calcification inhibitor and increasing the level of phosphate. ${ }^{2,3}$ Thus, serum ALP level has been used to diagnose hepatic or bony disease, ${ }^{\mathbf{4} 5}$ and is related to vascular calcification. ${ }^{6}$

Because vascular calcification increases cardiovascular risk, ALP level may be related to cardiovascular risk; indeed, the relation was first shown in patients with kidney failure. ALP level is significantly associated with cardiovascular mortality in hemodialysis patients. ${ }^{7-9}$ Furthermore, higher ALP is related to adverse outcome among survivors of myocardial infarction (MI) ${ }^{10}$ or stroke. ${ }^{11}$

In a recent study, higher ALP predicted mortality, MI, and stent thrombosis after percutaneous coronary intervention (PCI) with a drug-eluting stent (DES). ${ }^{12}$ Considering that vascular calcification hampers the process of re-endothelialization, ALP may contribute to stent thrombosis after PCI whereas coronary artery bypass grafting $(\mathrm{CABG})$ uses the endothelialized vein or artery for the graft. In addition, the anti-inflammatory effects of ALP may be beneficial for cardiac surgery patients by modulating the development of perioperative inflammatory responses. ${ }^{13-15}$ In a previous study, low postoperative ALP level was associated with worse clinical outcome in infants undergoing cardiothoracic surgery. ${ }^{16}$ Therefore, the relationship between ALP and adverse outcome in CABG patients may differ from that in PCI patients. If so, then ALP level could be used to determine the appropriate revascularization technique. No previous study, however, has investigated the relationship between preoperative ALP and postoperative adverse outcomes of CABG. In this study, we therefore retrospectively evaluated the relationship between serum ALP and major adverse cardiac events (MACE) after off-pump coronary artery bypass surgery (OPCAB).

\section{Methods}

Subjects

This study was approved by the Institutional Review Board of Seoul National University Hospital (No. H-1316080-498) and was conducted in accordance with the Declaration of Helsinki. This retrospective study did not require

Received December 15, 2016; revised manuscript received January 16, 2017; accepted February 3, 2017; released online March 2, 2017 Time for primary review: 27 days

Department of Anesthesiology and Pain Medicine (T.K.K., D.M.H., Y.H.C., C.-H.K., Y.J.C., Y.J.), Department of Internal Medicine and Cardiovascular Center (J.-B.P., K.W.P., H.-S.K.), Seoul National University Hospital, Seoul, Korea

Mailing address: Yunseok Jeon, MD, PhD, Department of Anesthesiology and Pain Medicine, Seoul National University Hospital, Daehakro 101, Seoul, Korea, 03080. E-mail: jeonyunseok@gmail.com

ISSN-1346-9843 All rights are reserved to the Japanese Circulation Society. For permissions, please e-mail: cj@j-circ.or.jp 


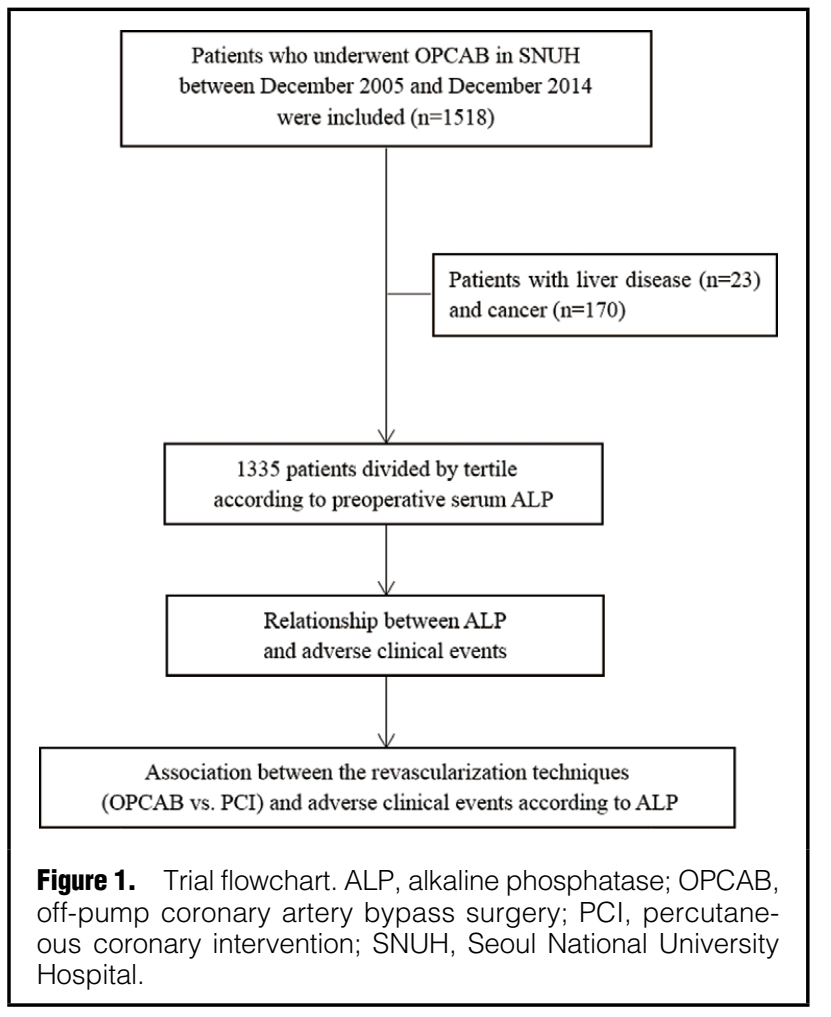

individual consent, based on the institutional guidelines for waiving consent. We retrospectively included patients who underwent OPCAB between December 2005 and December 2014 at Seoul National University Hospital. Initially, we enrolled a total of 1,518 patients. We excluded patients with established liver disease $(n=23)$ and cancer $(n=170)$ at the time of surgery because these are known to affect both ALP level and adverse outcome. Liver disease was defined as medical record confirmed diagnosis of liver abscess, cirrhosis, hepatitis, jaundice, and necrosis of the liver. Previously diagnosed cancer patients were identified by reviewing medical records. Ultimately, 1,335 patients were enrolled (Figure 1). To compare the outcomes of PCI with DES, we used the previously published data of Park et al. ${ }^{12}$ Their study protocol was approved by the Institutional Review Board of Seoul National University Hospital (No. H-1108099-374), and all patients gave written informed consent for registry enrollment and the retrospective analysis of their clinical data. They prospectively enrolled patients who underwent PCI with DES between February 2003 and June 2006 at the present center, and excluded patients with liver disease and cancer. These data included a total of 1,636 patients who had recorded serum ALP before PCI with DES.

\section{CABG and Follow-up}

Treatment during OPCAB and the hospitalization procedures were standardized according to institutional protocol. Surgery and anesthesia were performed by a single surgeon and 2 anesthesiologists (Y.J. or D.M.H.). Briefly, OPCAB was performed through a median sternotomy without cardiopulmonary bypass. If severe hemodynamic instability occurred during coronary anastomosis, intra-aortic balloon pump or extracorporeal support was started intraoperatively. The left internal thoracic artery and the greater saphenous vein were used as the bypass conduits. Angiographic follow-up was performed on the first postoperative day to check graft patency, and aspirin therapy was continued. A postoperative antiplatelet agent such as ticlopidine or clopidogrel was prescribed for at least 4 weeks from the first postoperative day. All patients were reevaluated at 1,3 , and 6 months after surgery. Routine angiographic follow-up was performed 1 year after surgery.

\section{Patient Data}

The electronic medical records of enrolled patients who underwent OPCAB were reviewed. We recorded demographic data, cardiovascular risk factors, medications, laboratory data, and intraoperative profiles. The data were collected by researchers blinded to ALP or were extracted automatically from the hospital database with the aid of the Institutional Medical Information Department. Intraoperative profile, including the number of anastomotic vessels, was collected from surgery and anesthesia records. Adverse events in patients transferred to another hospital or who did not respond to follow-up were ascertained via telephone interview. Cardiovascular risk factors included body mass index, smoking, diabetes mellitus, hypertension, hypercholesterolemia, peripheral vascular disease, stroke, prior MI, and CABG. To verify the accuracy of mortality data, we matched it to the nationwide official data on death certification offered by the National Statistical Office. We also obtained data from Park et al, ${ }^{12}$ which were used to analyze patients who underwent PCI.

\section{Outcomes and Definitions}

The primary outcome was the relationship between ALP and mortality in OPCAB patients, and the secondary outcomes were the relationships between ALP and cardiovascular mortality, MI, revascularization, and MACE. The effects of revascularization technique (OPCAB vs. PCI) on the relationship between ALP and mortality was also evaluated using OPCAB data from this study and PCI data from the Park et al study. ${ }^{\mathbf{1 2}}$ Mortality was classified into cardiovascular and non-cardiovascular. Cardiovascular mortality was defined as death due to MI, cardiac arrhythmia, heart failure, stroke, or vascular causes. Non-cardiovascular mortality was defined as death not caused by a cardiovascular problem. The diagnosis of MI was based on cardiac troponin $>10$-fold the upper reference limit, the development of new pathologic $Q$ waves, new persistent left bundle branch block, angiography-documented occlusion, or imaging evidence. ${ }^{17}$ Revascularization was defined as any intervention or surgery caused by an occlusion or thrombosis of the graft after OPCAB. Cardiologists confirmed the diagnosis of $\mathrm{MI}$ and coronary revascularization on review of hospital records. MACE was defined as the composite of allcause mortality, MI, and revascularization.

\section{Statistical Analysis}

To evaluate the relationship between ALP and mortality in OPCAB patients, patients were divided into 3 groups based on baseline ALP tertile (T1, <60 IU/L; T2, 60-76IU/L; T3, $>76 \mathrm{IU} / \mathrm{L})$. Continuous variables are presented as mean $\pm \mathrm{SD}$ and categorical variables as number and percentage. To compare baseline data between the tertiles, 1-way analysis of variance (ANOVA) or Kruskal-Wallis test was used for continuous variables and the chi-squared test or Fisher's exact test for categorical variables. Survival rates after OPCAB were examined on Kaplan-Meier analysis, and 


\begin{tabular}{|c|c|c|c|c|}
\hline \multirow[b]{3}{*}{ Demographics } & \multicolumn{3}{|c|}{ ALP tertile } & \multirow{2}{*}{$P$ value } \\
\hline & Lowest $(n=444)$ & Middle $(n=458)$ & Highest $(n=433)$ & \\
\hline & \\
\hline Age (years) & $65.5 \pm 10.1$ & $65.4 \pm 9.7$ & $66.0 \pm 9.3$ & 0.655 \\
\hline Male gender & $341(76.8)$ & $339(74.0)$ & $291(67.2)$ & 0.005 \\
\hline \multicolumn{5}{|l|}{ Risk factors } \\
\hline BMI $\left(\mathrm{kg} / \mathrm{m}^{2}\right)$ & $24.8 \pm 3.3$ & $24.5 \pm 2.8$ & $24.4 \pm 3.1$ & 0.106 \\
\hline Smoking & $196(44.4)$ & $219(47.8)$ & $193(44.7)$ & 0.525 \\
\hline Diabetes mellitus & $217(49.0)$ & $212(46.5)$ & $214(49.4)$ & 0.638 \\
\hline Hypertension & $312(70.4)$ & 338 (73.8) & 307 (70.9) & 0.476 \\
\hline Hypercholesterolemia & $118(26.6)$ & $126(27.5)$ & $126(29.2)$ & 0.687 \\
\hline Stroke & $67(15.1)$ & $74(16.2)$ & $62(14.3)$ & 0.745 \\
\hline Prior MI & $38(8.6)$ & $40(8.7)$ & $40(9.3)$ & 0.933 \\
\hline CKD & $52(11.7)$ & $61(13.3)$ & $73(16.9)$ & 0.080 \\
\hline Prior CABG & $8(1.8)$ & $21(4.6)$ & $19(4.4)$ & 0.045 \\
\hline Primary diagnosis of Ml at admission & $65(14.6)$ & $78(17.0)$ & $76(17.6)$ & 0.460 \\
\hline \multicolumn{5}{|l|}{ Laboratory variables } \\
\hline $\operatorname{LVEF}(\%)$ & $54.8 \pm 12.4$ & $54.4 \pm 13.2$ & $52.1 \pm 13.7$ & 0.004 \\
\hline $\operatorname{GFR}\left(\mathrm{mL} / \mathrm{min} / 1.73 \mathrm{~m}^{2}\right)$ & $67.8 \pm 21.4$ & $66.9 \pm 23.0$ & $64.8 \pm 25.9$ & 0.155 \\
\hline hs-CRP (mg/L) & $0.86 \pm 2.29$ & $0.87 \pm 1.82$ & $1.34 \pm 3.12$ & 0.005 \\
\hline Hemoglobin (g/dL) & $12.8 \pm 1.6$ & $12.9 \pm 1.7$ & $12.7 \pm 1.8$ & 0.267 \\
\hline $\mathrm{HDL}-\mathrm{C}(\mathrm{mg} / \mathrm{dL})$ & $41.6 \pm 10.6$ & $42.3 \pm 11.2$ & $41.0 \pm 10.8$ & 0.230 \\
\hline LDL-C (mg/dL) & $90.5 \pm 31.6$ & $97.1 \pm 38.7$ & $98.1 \pm 38.4$ & 0.005 \\
\hline Calcium (mg/dL) & $8.9 \pm 0.6$ & $9.1 \pm 2.9$ & $9.0 \pm 0.5$ & 0.221 \\
\hline Phosphorus (mg/dL) & $3.5 \pm 0.7$ & $3.4 \pm 0.6$ & $3.4 \pm 0.7$ & 0.504 \\
\hline Albumin $(\mathrm{g} / \mathrm{dL})$ & $4.0 \pm 0.5$ & $4.0 \pm 0.4$ & $3.9 \pm 0.5$ & 0.176 \\
\hline Bilirubin (mg/dL) & $0.77 \pm 0.40$ & $0.77 \pm 0.48$ & $0.72 \pm 0.41$ & 0.067 \\
\hline SGOT (IU/L) & $28.5 \pm 21.7$ & $28.3 \pm 21.1$ & $29.0 \pm 20.5$ & 0.885 \\
\hline SGPT (IU/L) & $27.4 \pm 23.9$ & $27.5 \pm 21.5$ & $30.5 \pm 40.6$ & 0.227 \\
\hline ALP (IU/L) & $49.2 \pm 7.4$ & $67.6 \pm 4.7$ & $97.8 \pm 23.2$ & $<0.001$ \\
\hline \multicolumn{5}{|l|}{ Surgical data } \\
\hline Multi-vessel disease & $506(96.0)$ & 397 (96.4) & $376(95.0)$ & 0.579 \\
\hline No. grafts & $3(1-5)$ & $3(1-6)$ & $3(1-6)$ & 0.190 \\
\hline
\end{tabular}

Data are presented as mean \pm SD, median (range), or $n(\%)$. ACEI, angiotensin-converting enzyme inhibitor; ALP, alkaline phosphatase; BMI, body mass index; CABG, coronary-artery bypass grafting; CKD, chronic kidney disease; GFR, glomerular filtration rate; HDL-C, high-density lipoprotein cholesterol; hs-CRP, high-sensitivity C-reactive protein; LDL-C, low-density lipoprotein cholesterol; LVEF, left ventricular ejection fraction; MI, myocardial infarction; OPCAB, off-pump coronary artery bypass surgery; SGOT, serum glutamic oxaloacetic transaminase; SGPT, serum glutamate-pyruvate transaminase.

differences in survival rate according to ALP tertile were compared using log-rank test.

After checking for violation of the proportional hazard assumption, Cox proportional hazards regression models were used to calculate the HR and $95 \%$ CI for each factor. The lowest preoperative ALP tertile was used as a reference. HR was adjusted for covariates related to ALP level or cardiovascular outcome. We constructed 2 models with baseline characteristics (model 1) or adjusted additional cardiovascular risk factors (model 2). A linear trend was assumed for continuous variables, and a Cox regression model was confirmed using restricted cubic splines. For categorical variables, proportional hazards assumption was verified with log-log survival plots.

To determine whether $\mathrm{C}$-reactive protein (CRP) provides additional information in predicting adverse outcome, Cox regression analysis was carried out after dividing patients into quartiles according to cut-offs of ALP and CRP, which were obtained using the minimum P-value approach.

To evaluate the influence of the revascularization tech- nique on adverse clinical events, the PCI patient data from a previous study ${ }^{12}$ were compared with those of the OPCAB patients. Enrolled patients were divided into tertiles according to baseline ALP (T1, <61 IU/L; T2, 61-77 IU/L; T3, $>77 \mathrm{IU} / \mathrm{L})$. Using the Cox proportional hazards regression model, associations between ALP and adverse events were evaluated.

$\mathrm{P}<0.05$ was considered to indicate statistical significance. Analysis were performed using SPSS 18.0 (SPSS, Chicago, IL, USA) and SAS 9.2 (SAS Institute, Cary, NC, USA).

\section{Results}

\section{Demographic Data}

Patients who underwent OPCAB were divided into tertiles (lowest tertile, ALP <60 IU/L, n=444; middle tertile, ALP 60-76IU/L, n=458; highest tertile, ALP >76IU/L, n=433). ALP ranged from 25 to $230 \mathrm{IU} / \mathrm{L}$ (mean, $71.5 \pm 24.4 \mathrm{IU} / \mathrm{L}$ ). Demographic and clinical subject characteristics according 


\begin{tabular}{|c|c|c|c|c|c|c|c|}
\hline & \multirow{2}{*}{ Events $\mathrm{n}(\%)$} & \multicolumn{3}{|c|}{ Model 1} & \multicolumn{3}{|c|}{ Model 2} \\
\hline & & HR & $95 \% \mathrm{Cl}$ & $P$ value & HR & $95 \% \mathrm{Cl}$ & $P$ value \\
\hline \multicolumn{8}{|c|}{ All-cause mortality } \\
\hline Lowest tertile & $63(14.2)$ & 1 & & & 1 & & \\
\hline Middle tertile & $76(16.7)$ & 0.997 & $0.708-1.404$ & 0.986 & 0.882 & $0.592-1.314$ & 0.537 \\
\hline Highest tertile & $78(18.1)$ & 1.171 & $0.834-1.643$ & 0.363 & 0.915 & $0.605-1.383$ & 0.672 \\
\hline \multicolumn{8}{|c|}{ Cardiovascular mortality } \\
\hline Lowest tertile & $32(7.2)$ & 1 & & & 1 & & \\
\hline Middle tertile & $30(6.6)$ & 0.774 & $0.466-1.286$ & 0.3236 & 0.698 & $0.383-1.272$ & 0.240 \\
\hline Highest tertile & $41(9.5)$ & 1.227 & $0.769-1.956$ & 0.3904 & 0.959 & $0.540-1.703$ & 0.886 \\
\hline \multicolumn{8}{|l|}{ MI } \\
\hline Lowest tertile & $14(3.2)$ & 1 & & & 1 & & \\
\hline Middle tertile & $18(4)$ & 1.133 & $0.557-2.305$ & 0.7306 & 0.945 & $0.422-2.112$ & 0.890 \\
\hline Highest tertile & $22(5.1)$ & 1.541 & $0.784-3.029$ & 0.2095 & 0.940 & $0.413-2.139$ & 0.883 \\
\hline \multicolumn{8}{|c|}{ Revascularization } \\
\hline Lowest tertile & $27(6.1)$ & 1 & & & 1 & & \\
\hline Middle tertile & $26(5.7)$ & 0.914 & $0.532-1.567$ & 0.7427 & 1.231 & $0.669-2.263$ & 0.504 \\
\hline Highest tertile & $18(4.2)$ & 0.640 & $0.350-1.170$ & 0.1470 & 0.650 & $0.319-1.322$ & 0.234 \\
\hline \multicolumn{8}{|l|}{ MACE } \\
\hline Lowest tertile & $91(20.5)$ & 1 & & & 1 & & \\
\hline Middle tertile & $100(21.9)$ & 0.983 & $0.737-1.310$ & 0.9049 & 0.997 & $0.718-1.385$ & 0.987 \\
\hline Highest tertile & 96 (22.2) & 1.039 & $0.776-1.391$ & 0.7981 & 0.915 & $0.650-1.290$ & 0.613 \\
\hline
\end{tabular}

Model 1, HR adjusted for age, sex, hypertension, diabetes mellitus, and primary diagnosis of Ml; model 2, HR adjusted for model 1 variables and additional covariates as follows: (1) traditional risk factors including smoking, dyslipidemia, use of aspirin, clopidogrel, $\beta$-blockers, ACEls, and LVEF; (2) angiographic data including multi-vessel disease, and number of grafts; and (3) laboratory data including GFR, hs-CRP, hemoglobin, HDL-C, bilirubin, aspartate aminotransferase, alanine aminotransferase, albumin, calcium, phosphorus. MACE, major adverse cardiac events. Other abbreviations as in Table 1.

A

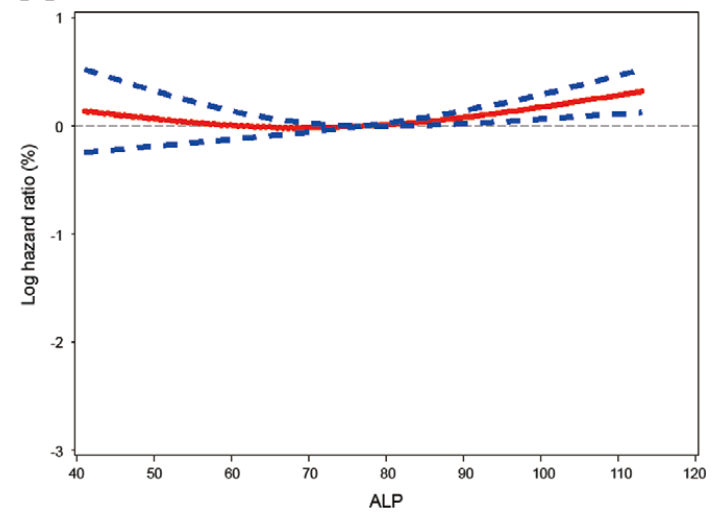

B

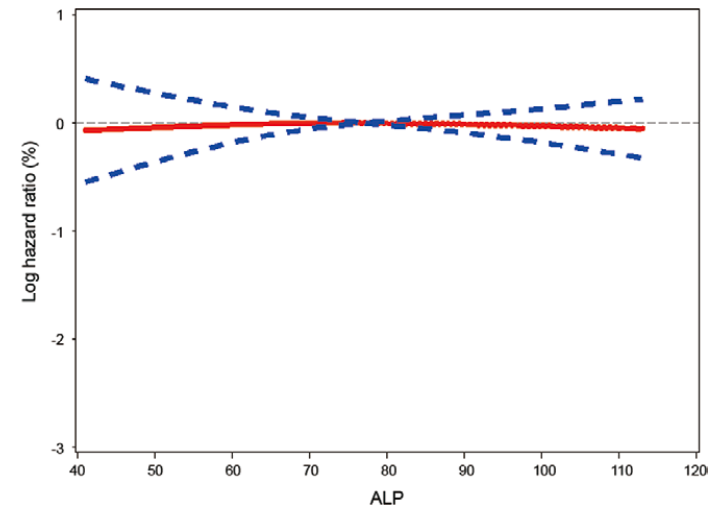

Figure 2. Restricted cubic spline regression model of the HR for all-cause mortality according to alkaline phosphatase (ALP) after off-pump coronary artery bypass surgery. (A) Unadjusted and (B) adjusted by the variables in model 2, Table 2. Solid line, HR; dotted lines, 95\% Cl.

to ALP tertile are listed in Table 1. Patients in the highest tertile tended to have higher CRP and lower left ventricular ejection fraction. Of the 1,335 patients who underwent OPCAB, $8(0.6 \%)$ required conversion from off-pump to on-pump CABG due to severe hemodynamic instability.

\section{Adverse Clinical Events}

The median follow-up duration was 31 months (IQR, 14-53 months). Postoperative MACE occurred in 21.6\% of patients $(288 / 1,335)$ who underwent OPCAB. Postop- erative MACE consisted of all-cause mortality, $n=217$; cardiovascular mortality, $n=103 ; \mathrm{MI}, \mathrm{n}=54$; and revascularization, $\mathrm{n}=71$ (Table 2).

When restricted cubic spline regression was used to evaluate the adjusted association between ALP and all-cause mortality, the HR of mortality remained constant as preoperative ALP increased (model 2, adjusted HR, 0.995; 95\% CI: $0.935-1.059, \mathrm{P}=0.862$, Figure 2). This was in contrast to the previous PCI study, in which the HR of mortality increased linearly as ALP increased (model 2, adjusted 
A

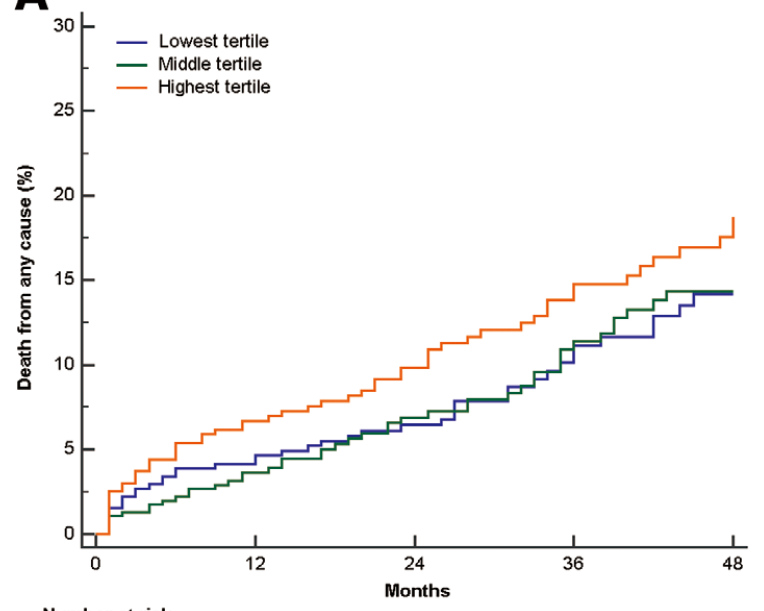

Number at risk

$\begin{array}{lllll}\begin{array}{c}\text { Lowest tertIle } \\ 444\end{array} & 357 & 268 & 172 & 115 \\ \begin{array}{c}\text { Middle tertile } \\ 458\end{array} & 364 & 270 & 193 & 145 \\ \begin{array}{c}\text { Highest tertile } \\ 433\end{array} & 327 & 254 & 175 & 132\end{array}$

B

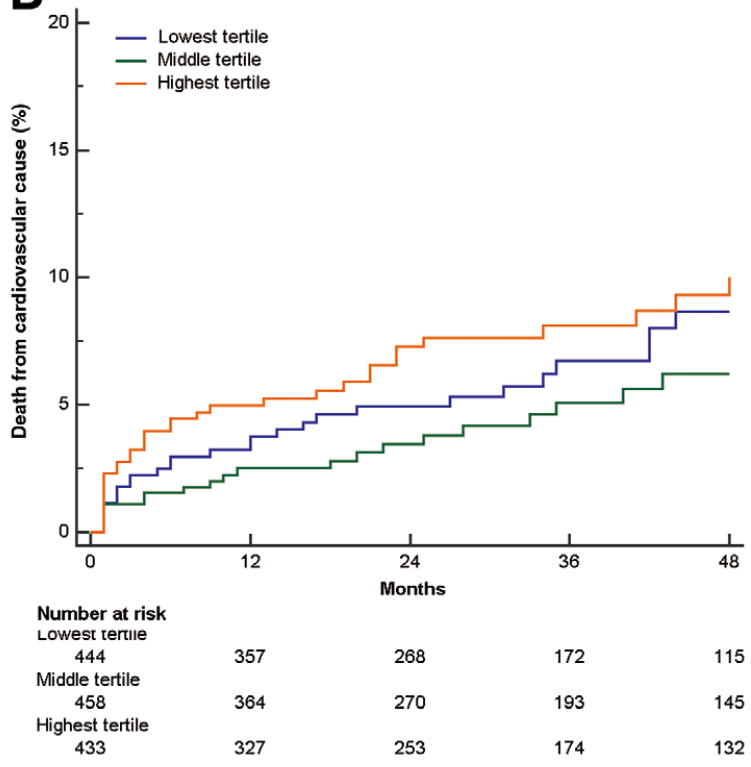

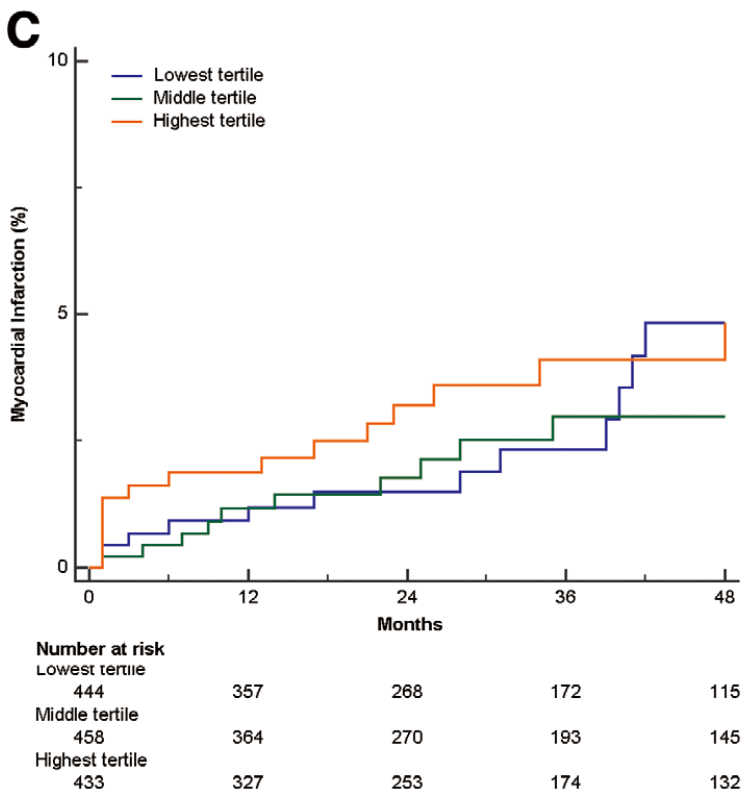

Figure 3. Kaplan-Meier time-to-event curves for (A) all-cause mortality, (B) cardiovascular mortality, and (C) myocardial infarction vs. preoperative alkaline phosphatase (ALP) tertile following off-pump coronary artery bypass surgery. MACE, major adverse cardiac event.

HR, 1.25; 95\% CI: 1.16-1.36, P<0.001, Figure S1). On Kaplan-Meier time-to-event curves, all-cause mortality was not significantly different between the ALP tertiles (Figure 3). Similar results were found for cardiovascular mortality, MI, revascularization, and MACE. According to the Cox proportional hazards regression analysis, there was no association between ALP tertile and all-cause mortality after adjusting for confounders. The adjusted $\mathrm{HR}$ for all-cause mortality was 0.882 (95\% CI: 0.592-1.314, $\mathrm{P}=0.537)$ for the middle tertile and 0.915 (95\% CI: $0.605-$ 1.383, $\mathrm{P}=0.672$ ) for the highest tertile (model 2, Table 2). Similarly, there were no associations between ALP tertile and cardiovascular mortality, MI, revascularization, or MACE in the OPCAB patients.

On multivariate-adjusted Cox regression analysis, the high ALP-high CRP group was at a higher risk for allcause mortality compared with the low ALP-low CRP group (adjusted HR, 1.935 ; 95\% CI: 1.280-2.923, $\mathrm{P}=0.002$, Table S1).

\section{Outcomes of OPCAB vs. PCI}

Cox proportional hazards regression analysis was used to evaluate potential associations between revascularization technique (OPCAB vs. PCI) and adverse clinical events 
(Figure S2). After adjusting for baseline characteristics, the HR for all-cause mortality in the lowest tertile was 2.604 (model 1, 95\% CI: $1.447-4.685, \mathrm{P}=0.001$ ), in favor of PCI (Table S2). When adjusted for additional cardiovascular risk factors, the pattern was similar (model 2, HR, 2.822; 95\% CI: $1.352-5.886, \mathrm{P}=0.008$ ). In the highest ALP tertile, however, the adjusted HR was lower in the OPCAB than in the PCI group, without statistical significance (model 1, HR, 0.731; 95\% CI: 0.514-1.039, $\mathrm{P}=0.081$ ).

\section{Discussion}

In the present study high serum preoperative ALP was not related to adverse clinical outcomes such as mortality, MI, or revascularization after OPCAB. As ALP increased, the hazard of mortality remained constant after adjusting for confounders. Similar results were found for cardiovascular mortality, MI, revascularization, and MACE. This is in contrast to a previous study that showed an association between high ALP and poor clinical outcome after PCI.

It has been suggested that high ALP is related to adverse cardiovascular outcomes. The adverse effects of ALP on the vascular system may originate from accelerated vascular calcification, in which ALP is believed to be an indicator or a regulator. ${ }^{\mathbf{1 8 , 1 9}}$ Vascular calcification is an actively regulated process orchestrated by vascular smooth muscle cells and bone matrix proteins. Moreover, osteogenic factors including ALP are upregulated in calcified vessels. ${ }^{20} \mathrm{It}$ is also known that ALP controls vascular calcification by the hydrolysis of pyrophosphate, a potent inhibitor of vascular calcification. ${ }^{\mathbf{6} 21}$ Vascular calcification is associated with vascular aging and atherosclerosis, which impairs cardiovascular hemodynamics, resulting in substantial morbidity and mortality. ${ }^{22-24}$ Considering that ALP is related to vascular calcification, increased ALP could be expected to be associated with adverse outcomes of vascular disease.

A recent study showed that increased ALP is an independent predictor of mortality after stroke.11 That study examined 2,029 participants with acute stroke and showed that higher ALP was associated with both all-cause mortality and vascular mortality in either ischemic or hemorrhagic stroke. Moreover, Park et al reported that higher ALP was an independent predictor of mortality, MI, and stent thrombosis in 1,636 patients who underwent PCI.12 Considering that OPCAB patients have similar cardiovascular risk factors as PCI patients, vascular calcification may affect adverse outcomes after OPCAB. Few studies, however, have evaluated the impact of vascular calcification on OPCAB outcome. A recent study reported an association between severe coronary calcification and worse outcome after CABG, including 1-year MACE, death, or MI. ${ }^{25}$ The authors suggested that calcium deposition could lead to distal endothelial dysfunction and embolic phenomenon, which may lead to ischemic insult, or that the presence of coronary calcification could reflect systemic atherosclerotic disease. Their results also raise the possibility that ALP may be associated with CABG outcome through the acceleration of vascular calcification.

In contrast, however, with the previous study on PCI, high ALP was were not related to adverse clinical outcome after OPCAB in the present study. One possible reason for the association between ALP and poor clinical outcome of PCI is that coronary calcification hampers the process of re-endothelialization. Given that endothelialized artery or vein grafts are used for revascularization during CABG, vascular calcification may affect the adverse outcomes of CABG differently. We did not, however, assess vascular endothelialization, and further studies are needed to evaluate the underlying mechanism. ALP, meanwhile, functions as part of the host defense against inflammation, in part due to its ability to neutralize endotoxin. ${ }^{13-15}$ A recent study reported that low ALP following cardiothoracic surgery in infants is independently associated with increased vasoactive/inotropic requirements, intubation time, and length of hospital stay. ${ }^{16}$ Thus, vascular effects of ALP on clinical outcome may be offset by its protective effects in the attenuation of inflammatory reactions following OPCAB. ${ }^{14}$

Using the Park et al data, ${ }^{12}$ we evaluated the potential association between revascularization technique (OPCAB vs. PCI) and adverse outcome according to ALP level. After adjustments, the HR for mortality in OPCAB compared with PCI decreased as ALP increased (Figure S2). The current guidelines support the use of CABG over PCI for left main disease or 3-vessel disease with a high SYNTAX score, which is based on the location, severity, and extent of coronary stenosis. ${ }^{26}$ Although the SYNTAX score provides additional points in the presence of calcification, it includes only heavy calcification, and angiography does not fully represent the coronary calcification. ${ }^{25}$ Considering the independent association between ALP and coronary calcification, ${ }^{12}$ ALP score might be beneficial for predicting coronary risk and determining the optimal revascularization technique. Further prospective studies, however, are needed to obtain more information on the association between ALP and selection of revascularization technique.

Several studies have shown the prognostic value of preoperative CRP for postoperative outcome. ${ }^{27,28}$ Park et al showed that the combined evaluation of ALP and CRP was more effective at predicting adverse outcome than the measurement of either marker alone in PCI patients. ${ }^{\mathbf{1 2}}$ In the present OPCAB patients, the high ALP-high CRP group was at a higher risk for all-cause mortality compared with the low ALP-low CRP group. The adjusted HR in the high ALP-high CRP group, however, was much smaller than in the PCI group.

This study had some limitations. First, it was a retrospective study. Thus, we did not collect prospectively patient information or cardiac events in the OPCAB patients. Also, the data obtained from OPCAB and PCI patients were retrospectively analyzed, not randomized. Although we adjusted for multiple confounding factors associated with ALP and adverse outcome, the hidden biases could have considerably affected the analysis and we cannot rule out possible residual confounders. Likewise, a prospective randomized trial with a larger sample size may be needed to show the relationship between ALP and procedure outcomes more relevantly. Second, the OPCAB procedures included in this study were performed at a single center by a single surgeon. In most CABG patients, surgery was performed without cardiopulmonary bypass, therefore this result might not be directly applicable to conventional $\mathrm{CABG}$, which is performed with cardiopulmonary bypass. Third, because this was a retrospective study, we did not have information on parathyroid hormone or dietary vitamin D intake, which are known to be related to ALP level. Although ALP has been shown to be associated with mortality independently of parathyroid hormone and vitamin $\mathrm{D},{ }^{\mathbf{8}}$ these data may have been helpful while interpreting the study results. 


\section{Conclusions}

Unlike for PCI, high serum preoperative ALP is not associated with adverse clinical events such as mortality, MI, or revascularization after OPCAB. Therefore, ALP might be considered when determining the optimal revascularization technique.

\section{Acknowledgments}

We thank the Medical Research Collaborating Center of Seoul National University Hospital (Seoul, Korea) for the assistance with statistics and supervision.

\section{Disclosures}

The authors declare no conflict of interest.

\section{References}

1. Weiss MJ, Cole DE, Ray K, Whyte MP, Lafferty MA, Mulivor $\mathrm{RA}$, et al. A missense mutation in the human liver/bone/kidney alkaline phosphatase gene causing a lethal form of hypophosphatasia. Proc Natl Acad Sci USA 1988; 85: 7666-7669.

2. Harmey D, Hessle L, Narisawa S, Johnson KA, Terkeltaub R, Millan JL. Concerted regulation of inorganic pyrophosphate and osteopontin by akp2, enpp1, and ank: An integrated model of the pathogenesis of mineralization disorders. Am J Pathol 2004; 164: $1199-1209$

3. Schoppet M, Shanahan CM. Role for alkaline phosphatase as an inducer of vascular calcification in renal failure? Kidney Int 2008; 73: $989-991$

4. Suzuki N, Irie M, Iwata K, Nakane H, Yoshikane M, Koyama $\mathrm{Y}$, et al. Altered expression of alkaline phosphatase (ALP) in the liver of primary biliary cirrhosis (PBC) patients. Hepatol Res 2006; 35: 37-44.

5. Yorio MA, Sembaj A, Sanz E, Carriazo C, Moreno Barral J. Alkaline phosphatase isoenzymes for the diagnosis of metastatic tumors and lymphomas of liver and bone. Medicina 2000; 60: $311-315$

6. Lomashvili KA, Garg P, Narisawa S, Millan JL, O'Neill WC. Upregulation of alkaline phosphatase and pyrophosphate hydrolysis: Potential mechanism for uremic vascular calcification. Kidney Int 2008; 73: 1024-1030.

7. Block GA, Raggi P, Bellasi A, Kooienga L, Spiegel DM. Mortality effect of coronary calcification and phosphate binder choice in incident hemodialysis patients. Kidney Int 2007; 71: 438-441.

8. Regidor DL, Kovesdy CP, Mehrotra R, Rambod M, Jing J, McAllister CJ, et al. Serum alkaline phosphatase predicts mortality among maintenance hemodialysis patients. $J$ Am Soc Nephrol 2008; 19: 2193-2203.

9. Slinin Y, Foley RN, Collins AJ. Calcium, phosphorus, parathyroid hormone, and cardiovascular disease in hemodialysis patients: The USRDS waves 1, 3, and 4 study. J Am Soc Nephrol 2005; 16: $1788-1793$.

10. Tonelli M, Curhan G, Pfeffer M, Sacks F, Thadhani R, Melamed ML, et al. Relation between alkaline phosphatase, serum phosphate, and all-cause or cardiovascular mortality. Circulation 2009; 120: $1784-1792$.

11. Ryu WS, Lee SH, Kim CK, Kim BJ, Yoon BW. Increased serum alkaline phosphatase as a predictor of long-term mortality after stroke. Neurology 2010; 75: 1995-2002.

12. Park JB, Kang DY, Yang HM, Cho HJ, Park KW, Lee HY, et al. Serum alkaline phosphatase is a predictor of mortality, myocardial infarction, or stent thrombosis after implantation of coronary drug-eluting stent. Eur Heart J 2013; 34: 920-931.

13. Kats S, Brands R, Seinen W, de Jager W, Bekker MW, Hamad MA, et al. Anti-inflammatory effects of alkaline phosphatase in coronary artery bypass surgery with cardiopulmonary bypass. Recent Pat Inflamm Allergy Drug Discov 2009; 3: 214-220.

14. Kats S, Schonberger JP, Brands R, Seinen W, van Oeveren W. Endotoxin release in cardiac surgery with cardiopulmonary bypass: Pathophysiology and possible therapeutic strategies. An update. Eur J Cardiothorac Surg 2011; 39: 451-458.
15. Verweij WR, Bentala H, Huizinga-van der Vlag A, Miek van Loenen-Weemaes A, Kooi K, Meijer DK, et al. Protection against an Escherichia coli-induced sepsis by alkaline phosphatase in mice. Shock 2004; 22: 174-179.

16. Davidson J, Tong S, Hauck A, Lawson DS, Jaggers J, Kaufman $\mathrm{J}$, et al. Alkaline phosphatase activity after cardiothoracic surgery in infants and correlation with post-operative support and inflammation: A prospective cohort study. Crit Care 2012; 16: R160.

17. Thygesen K, Alpert JS, Jaffe AS, Simoons ML, Chaitman BR, White HD, et al. Third universal definition of myocardial infarction. Eur Heart J 2012; 33: 2551-2567.

18. O'Neill WC, Sigrist MK, McIntyre CW. Plasma pyrophosphate and vascular calcification in chronic kidney disease. Nephrol Dial Transplant 2010; 25: 187-191.

19. Johnson RC, Leopold JA, Loscalzo J. Vascular calcification: Pathobiological mechanisms and clinical implications. Circ Res 2006; 99: 1044-1059.

20. Shanahan CM, Cary NR, Salisbury JR, Proudfoot D, Weissberg PL, Edmonds ME. Medial localization of mineralization-regulating proteins in association with Monckeberg's sclerosis: Evidence for smooth muscle cell-mediated vascular calcification. Circulation 1999; 100: 2168-2176.

21. Moss DW, Eaton RH, Smith JK, Whitby LG. Association of inorganic-pyrophosphatase activity with human alkaline-phosphatase preparations. Biochem J 1967; 102: 53-57.

22. Arad Y, Spadaro LA, Goodman K, Newstein D, Guerci AD. Prediction of coronary events with electron beam computed tomography. J Am Coll Cardiol 2000; 36: 1253-1260.

23. Demer LL, Tintut Y. Vascular calcification: Pathobiology of a multifaceted disease. Circulation 2008; 117: 2938-2948.

24. Wayhs R, Zelinger A, Raggi P. High coronary artery calcium scores pose an extremely elevated risk for hard events. J Am Coll Cardiol 2002; 39: 225-230.

25. Ertelt K, Genereux P, Mintz GS, Reiss GR, Kirtane AJ, Madhavan $\mathrm{MV}$, et al. Impact of the severity of coronary artery calcification on clinical events in patients undergoing coronary artery bypass grafting (from the Acute Catheterization and Urgent Intervention Triage Strategy Trial). Am J Cardiol 2013; 112: 1730-1737.

26. Windecker S, Kolh P, Alfonso F, Collet JP, Cremer J, Falk V, et al. 2014 ESC/EACTS Guidelines on myocardial revascularization: The Task Force on Myocardial Revascularization of the European Society of Cardiology (ESC) and the European Association for Cardio-Thoracic Surgery (EACTS), Developed with the special contribution of the European Association of Percutaneous Cardiovascular Interventions (EAPCI). Eur Heart $J$ 2014; 35: $2541-2619$

27. Kangasniemi OP, Biancari F, Luukkonen J, Vuorisalo S, Satta $\mathrm{J}$, Pokela R, et al. Preoperative C-reactive protein is predictive of long-term outcome after coronary artery bypass surgery. Eur $J$ Cardiothorac Surg 2006; 29: 983-985.

28. Perry TE, Muehlschlegel JD, Liu KY, Fox AA, Collard CD, Body SC, et al. Preoperative C-reactive protein predicts longterm mortality and hospital length of stay after primary, nonemergent coronary artery bypass grafting. Anesthesiology 2010; 112: $607-613$.

\section{Supplementary Files}

\section{Supplementary File 1}

Figure S1. Restricted cubic spline regression model of the HR for all-cause mortality according to alkaline phosphatase (ALP) after percutaneous coronary intervention.

Figure S2. Adjusted HR for off-pump coronary artery bypass surgery compared with percutaneous coronary intervention for allcause mortality: (A) model 1 adjusted; (B) model 2 adjusted.

Table S1. Multivariate indicators of all-cause mortality in OPCAB patients

Table S2. Revascularization technique and adverse clinical outcomes vs. ALP

Please find supplementary file(s);

http://dx.doi.org/10.1253/circj.CJ-16-1288 